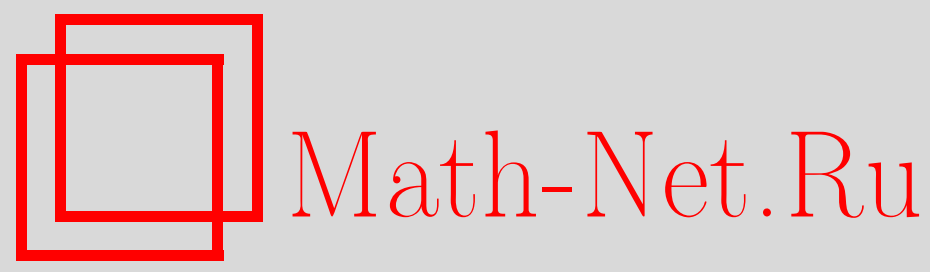

Ю. А. Будников, Асимптотическая верхняя оценка хроматического индекса случайных гиперграфов, Дискрет. матем., 2011, том 23, выпуск 3, 63-81

DOI: https://doi.org/10.4213/dm1153

Использование Общероссийского математического портала Math-Net.Ru подразумевает, что вы прочитали и согласны с пользовательским соглашением http://www . mathnet.ru/rus/agreement

Параметры загрузки:

IP : 54.164 .48 .24

26 апреля 2023 г., 13:49:36 
УДК 519.2

\title{
Асимптотическая верхняя оценка хроматического индекса случайных гиперграфов
}

\author{
() 2011 г. Ю. А. Будников
}

В работе предложена верхняя асимптотическая оценка хроматического индекса случайных гиперграфов для случая, когда длина ребра гиперграфа - возрастающая функция, зависящая от числа вершин гиперграфа.

Показано, что асимптотически с вероятностью 1 хроматический индекс $\chi(G)$ случайного однородного гиперграфа $G(n)$ не превосходит $c D(n) \log (k(n))$, где $n-$ число вершин $G(n), D(n)$ - математическое ожидание степени вершины $G(n), k(n)$ - число вершин на любом ребре $G(n), k(n)=o(n)$ при $n \rightarrow \infty, c>1-$ некоторая константа.

\section{1. Введение}

В статьях, посвященных теории гиперграфов, широко исследуются вопросы, относящиеся к оценке хроматического индекса гиперграфа. В [1] приводится доказательство верхней оценки хроматического индекса $\chi(G)$ любого однородного гиперграфа $G$, имеющего длину ребер $k$ и максимальную степень вершины $D$ :

$$
\chi(G) \leqslant(D-1) k+1 .
$$

Для детерминированных гиперграфов с фиксированной длиной ребра в [1] была доказана известная теорема об асимптотике хроматического индекса. Оказалось, что при ряде ограничений, наложенных на некоторые параметры асимптотически регулярного и однородного гиперграфа, его хроматический индекс ведет себя как степень вершины, если число вершин гиперграфа стремится к бесконечности.

Обобщение этого результата на случай, когда длина ребра гиперграфа растет с ростом числа вершин гиперграфа, невозможно. Доказательство этого факта представлено в [2], где приводится пример бесконечной подпоследовательности гиперграфов, хроматический индекс каждого из которых в точности равен его верхней оценке [1]

$$
\chi(G)=k(D-1)+1,
$$

где $k$ - длина ребра, $D$ - максимальная из степеней вершин гиперграфа. Поэтому теорему из [1] нельзя обобщить на случай, когда длина ребра растет с ростом числа вершин гиперграфа.

Тем не менее, можно построить несложные примеры последовательностей однородных и регулярных гиперграфов $G$, для которых $\chi(G)=D$. 
Если перейти к модели случайных гиперграфов, то при фиксированной длине ребра асимптотика их хроматического индекса получается напрямую из теоремы статьи [1], а в случае растущей длины ребра, как следует из работы [2], асимптотика хроматического индекса случайных гиперграфов может отличаться от полученной в [1].

В данной работе получена верхняя асимптотическая оценка хроматического индекса случайных гиперграфов для случая, когда длина ребра гиперграфа - некоторая возрастающая функция, зависящая от числа вершин гиперграфа.

Для удобства изложения введем обозначения

$$
\begin{aligned}
& f(n) \sim g(n) \quad \Longleftrightarrow \lim _{n \rightarrow \infty} \frac{f(n)}{g(n)}=1, \\
& f(n) \lesssim g(n) \quad \Longleftrightarrow \lim _{n \rightarrow \infty} \frac{f(n)}{g(n)} \leqslant 1, \\
& f(n) \gtrsim g(n) \quad \Longleftrightarrow \lim _{n \rightarrow \infty} \frac{f(n)}{g(n)} \geqslant 1, \\
& f(n)=o(g(n)) \Longleftrightarrow \lim _{n \rightarrow \infty} \frac{f(n)}{g(n)}=0 .
\end{aligned}
$$

Для формулировки основного результата необходимы следующие определения.

Упаковка в гиперграфе $G$ - это набор $P$ непересекающихся ребер $G$.

Хроматический индекс $\chi(G)$ графа $G$ - минимальное число упаковок, на которые можно разбить все ребра $G$. Рассмотрим полный гиперграф

$$
G(n)=(V(G(n)), E(G(n)))
$$

на $n$ вершинах, $k$-однородный, то есть любое его ребро $e \in E(G(n))$ содержит в точности $k$ вершин из множества $V(G(n))$. Степень любой его вершины есть $\left(\begin{array}{l}n-1 \\ k-1\end{array}\right)$, и $|E(G(n))|=\left(\begin{array}{l}n \\ k\end{array}\right)$.

Введем вероятностное пространство $(\Omega, \mathscr{F}, \mathbf{P})$, где $\Omega-$ множество всех подмножеств $Z_{i} \in E(G), i=1, \ldots, 2\left(\begin{array}{c}n \\ k\end{array}\right), \mathscr{F}-$ множество всевозможных подмножеств $\Omega$. Если происходит элементарный исход $Z_{i}=\left\{e_{i_{1}}, \ldots, e_{i_{m}}\right\}$, то говорим, что родились ребра $e_{i_{1}}, \ldots, e_{i_{m}}$. Пусть все ребра рождаются независимо с вероятностью $p(n)$. На самом деле имеется в виду стандартная вероятностная модель испытаний Бернулли, где элементарные события - это кортежи из 0, 1 длины $\left(\begin{array}{l}n \\ k\end{array}\right)$, где единицы на каких-то позициях соответствуют рожденным ребрам, а нули - нерожденным. На $\Omega$ вводится следующая вероятностная мера:

$$
\begin{aligned}
\mathbf{P}\left(Z_{i}\right) & =\mathbf{P}\left(\begin{array}{c}
\text { родилось множество ребер }\left\{e_{i_{1}}, \ldots, e_{i_{m}}\right\} \\
\text { и не родились все оставшиеся ребра } G(n)
\end{array}\right) \\
& =p(n)^{m}(1-p(n))^{\left(\begin{array}{l}
n \\
k
\end{array}\right)-m} .
\end{aligned}
$$

На этом вероятностном пространстве определяется случайная величина $G(n, p)$, принимающая значения $Z_{i}$ с вероятностями $\mathbf{P}\left(Z_{i}\right)$. Это и есть случайный гиперграф, хроматический индекс которого оценивается в данной работе.

Пусть

$$
p(n)=\frac{D(n)}{\left(\begin{array}{l}
n-1 \\
k-1
\end{array}\right)},
$$

где $D(n)$ - возрастающая функция от $n$. Далее для краткости будем опускать аргумент у функций $D(n), p(n), k(n)$, если это не будет приводить к неоднозначной трактовке. 


\section{2. Асимптотика числа родившихся упаковок}

Далее везде $c$ - произвольная константа, превосходящая 1. Длина ребра гиперграфа $k(n)-$ функция от числа вершин гиперграфа $n$, возрастающая с ростом $n$ в дискретном смысле [2].

Введем понятие случайной упаковки. Просто упаковка в детерминированном гиперграфе - это любое подмножество попарно непересекающихся ребер этого гиперграфа. Случайная упаковка - это упаковка в случайном гиперграфе $G(n, p)$. Она определяется следующим образом. Рассмотрим упаковку $P=\left\{e_{i_{1}}, \ldots, e_{i_{s}}\right\}$ полного гиперграфа на $n$ вершинах. Для каждого ее ребра $e_{i_{j}}, j=1, \ldots, s$ на вероятностном пространстве $\Omega$ можно определить индикаторную случайную величину $I\left(e_{i_{j}}\right)=1$ с вероятностью $p$, $I\left(e_{i_{j}}\right)=0$ с вероятностью $1-p$. Если $I\left(e_{i_{j}}\right)=1$, то говорим, что родилось ребро $e_{i_{j}}$, в противном случае говорим, что ребро $e_{i j}$ не родилось. По определению, случайной упаковкой случайного гиперграфа $G(n, p)$ называется случайная величина

$$
I(P)=\prod_{j=1}^{s} I\left(e_{i_{j}}\right)
$$

Пусть $M(n)$ - число упаковок размера $n /(k c)$ полного гиперграфа $G(n)$. В силу леммы 1 в [2],

$$
\begin{aligned}
M(n) & =\frac{n !}{(k !)^{n /(k c)}(n(1-1 / c)) !(n /(k c)) !} \\
& =\frac{\prod_{i=1}^{n / k c}\left(\begin{array}{c}
n-n / c+i k \\
k
\end{array}\right)}{(n /(k c)) !} .
\end{aligned}
$$

Пусть $P G(n)$ - множество упаковок размера $n /(k c)$ в $G(n)$. Естественным образом вводится понятие числа упаковок случайного гиперграфа $G(n, p)$ размера $n /(k c)$. Это есть случайная величина, равная сумме по всевозможным упаковкам $P$ размера $n /(k c)$ полного гиперграфа $G(n)$ индикаторных случайных величин $I(P)$, то есть $\sum_{P \in P G(n)} I(P)$. Подсчитаем ее математическое ожидание:

$$
\begin{aligned}
N(n) & =\mathbf{E}\left(\sum_{P \in P G(n)} I(P)\right)=M(n)\left(\frac{D}{\left(\begin{array}{c}
n-1 \\
k-1
\end{array}\right)}\right)^{n /(k c)} \\
& =\frac{n !}{(k !)^{n /(k c)}(n(1-1 / c)) !(n /(k c)) !}\left(\frac{D}{\left(\begin{array}{c}
n-1 \\
k-1
\end{array}\right)}\right)^{n /(k c)} .
\end{aligned}
$$

Теорема 1. Пусть $G(n, p(n))$ - случайньй гиперграф, $k(n)$ - возрастающая функция от $n$, причем $k(n)=o(n)$ при $n \rightarrow \infty$ и, начиная с некоторого $n$,

$$
p(n)=\frac{D(n)}{\left(\begin{array}{c}
n-1 \\
k(n)-1
\end{array}\right)} \leqslant 1, \quad D(n)>c\left(\frac{n}{k(n) c}\right)^{3}\left(\frac{c}{c-1}\right)^{k(n)} .
$$

Тогда для любого $\varepsilon>0$, начиная с некоторого $n$,

$$
\mathbf{P}\left(\left|\frac{1}{N(n)} \sum_{P \in P G(n)} I(P)-1\right|>\varepsilon\right)<\frac{1}{\varepsilon^{2}}\left(\frac{1}{N(n)}+\frac{1}{(n / k(n))(c /(c-1))^{k(n)}}\right) .
$$


Доказательство. В качестве основного инструмента используется неравенство Чебышёва:

$$
\mathbf{P}\left(\left|\frac{\sum_{P \in P G(n)} I(P)}{N(n)}-1\right|>\varepsilon\right) \leqslant \frac{\mathbf{D}\left(\sum_{P \in G(n)} I(P)\right)}{N^{2}(n) \varepsilon^{2}},
$$

где $\mathbf{D}(\xi)$ - дисперсия случайной величины $\xi$.

Далее рассматриваем упаковки $P$ в $G(n)$ только размера $n /(k c)$. Ясно, что

$$
\mathbf{D}\left(\sum_{P \in P G(n)} I(P)\right)=\sum_{P \in G(n)} \mathbf{D}(I(P))+2 \sum_{\substack{P_{i}, P_{j} \in G(n) \\ P_{i} \neq P_{j}}} \operatorname{cov}\left(I\left(P_{i}\right), I\left(P_{j}\right)\right),
$$

где $\operatorname{cov}(\xi, \eta)-$ ковариация случайных величин $\xi$ и $\eta$.

Поскольку $I(P)$ - индикаторная случайная величина, то при $n \rightarrow \infty$

$$
\begin{aligned}
\mathbf{D}(I(P)) & =\left(\frac{D}{\left(\begin{array}{c}
n-1 \\
k-1
\end{array}\right)}\right)^{n /(k c)}\left(1-\left(\frac{D}{\left(\begin{array}{l}
n-1 \\
k-1
\end{array}\right)}\right)^{n /(k c)}\right) \rightarrow \frac{1}{N^{2}(n) \varepsilon^{2}} \sum_{\substack{P \in P G(n) \\
|P|=n /(k c)}} \mathbf{D}(I(P)) \\
& =\frac{M(n)}{N^{2}(n) \varepsilon^{2}}\left(\frac{D}{\left(\begin{array}{c}
n-1 \\
k-1
\end{array}\right)}\right)^{n /(k c)}\left(1-\frac{D}{\left(\begin{array}{c}
n-1 \\
k-1
\end{array}\right)}\right)^{n /(k c)} \\
& =\frac{1}{N(n) \varepsilon^{2}}\left(1-\left(\frac{D}{\left(\begin{array}{c}
n-1 \\
k-1
\end{array}\right)}\right)^{n /(k c)}\right)<\frac{1}{N(n) \varepsilon^{2}} \rightarrow 0,
\end{aligned}
$$

потому что

$$
N(n)=M(n)\left(\frac{D}{\left(\begin{array}{c}
n-1 \\
k-1
\end{array}\right)}\right)^{n /(k c)}
$$

Пусть

$$
L(n)=\frac{1}{N^{2}(n) \varepsilon^{2}} \sum_{\substack{P_{i}, P_{j} \in P G(n) \\ P_{i} \neq P_{j}}} \operatorname{cov}\left(I\left(P_{i}\right), I\left(P_{j}\right)\right) .
$$

Для доказательства теоремы 1 осталось показать, что при $n \rightarrow \infty$

$$
L(n) \rightarrow 0
$$

Если рассмотреть сумму всевозможных ковариаций некоторой фиксированной случайной упаковки $I(P), P \in P G(n)$, и всех остальных случайных упаковок, то для каждой такой упаковки $I(P), P \in G(n)$, эта сумма будет одной и той же из соображений симметрии. Поэтому можно упростить выражение для суммы ковариаций следующим образом:

$$
R=\sum_{\substack{P_{i}, P_{j} \in P G(n) \\ P_{i} \neq P_{j}}} \operatorname{cov}\left(I\left(P_{i}\right), I\left(P_{j}\right)\right)=M(n) \sum_{P_{j} \in G(n)} \operatorname{cov}\left(I(P), I\left(P_{j}\right)\right),
$$

где $P$ - любая фиксированная упаковка размера $n /(k c)$ полного гиперграфа $G(n)$

Далее, выделим группы ковариаций в соответствии с числом общих ребер в упаковках $P$ и $P_{j}$ в $G(n)$. Для любого $i=1, \ldots, n /(k c)-1$ определим число упаковок $R(i)$ в $G(n)$, 
имеющих в точности $i$ ребер, которых нет в упаковке $P$. Это означает, что оставшиеся $n /(k c)-i$ ребер каждой такой упаковки совпадают с какими-то ребрами упаковки $P$.

Если упаковка $P_{j}$ имеет в точности $n /(k c)-i$ ребер, которые есть в упаковке $P$, то есть $i$ ее ребер не содержатся в упаковке $P$, то

$$
\operatorname{cov}\left(P, P_{j}\right)=\mathbf{E}\left(I(P) I\left(P_{j}\right)\right)-\mathbf{E}(I(P)) \mathbf{E}\left(I\left(P_{j}\right)\right)=\left(\frac{D}{\left(\begin{array}{c}
n-1 \\
k-1
\end{array}\right)}\right)^{n(k c)+i}-\left(\frac{D}{\left(\begin{array}{c}
n-1 \\
k-1
\end{array}\right)}\right)^{2 n /(k c)} .
$$

Замечание 1. Если $i=n /(k c)$, то есть все ребра упаковок $P$ и $P_{j}$ различны, то случайные величины $I(P)$ и $I\left(P_{j}\right)$ независимы и $\operatorname{cov}\left(P, P_{j}\right)=0$.

Если $i=0$, то $\operatorname{cov}\left(P, P_{j}\right)=\mathbf{D}(P)$, а сумма дисперсий уже оценена выше.

Поэтому можно перегруппировать $R$ следующим образом:

$$
R=\sum_{i=1}^{n /(k c)-1} R(i)\left(\left(\frac{D}{\left(\begin{array}{l}
n-1 \\
k-1
\end{array}\right)}\right)^{n /(k c)+i}-\left(\frac{D}{\left(\begin{array}{c}
n-1 \\
k-1
\end{array}\right)}\right)^{2 n /(k c)}\right)
$$

Предложение 1. Для $i=1, \ldots, n /(k c)-1$

$$
R(i)=\frac{1}{i !}\left(\begin{array}{c}
n /(k c) \\
i
\end{array}\right) \prod_{r=1}^{i}\left(\left(\begin{array}{c}
n-n / c+r k \\
k
\end{array}\right)-i\right) .
$$

Доказательство. Упаковка $P$ уже имеет $n /(k c)-i$ общих ребер с $P_{j}$. Надо добрать еще $i$ peбер.

Число способов выбрать из $n /(k c)$ ребер упаковки $P$ какие-то фиксированные $i$ ее ребер, по которым отличаются упаковка $P$ и другие упаковки $G(n)$ (то есть этих $i$ ребер в них нет, а все остальные ребра упаковки $P$ в них есть) равно $\left(\begin{array}{c}n /(k c) \\ i\end{array}\right)$. Из симметрии полного гиперграфа следует, что при любом выборе этих $i$ ребер получим одно и то же число упаковок $G(n)$, которые отличаются от упаковки $P$ по этим $i$ ребрам.

Число способов выбрать $r$-е ребро упаковки $P_{j}$, которая уже имеет $n /(k c)-i$ общих ребер с $P$ равно $\left(\begin{array}{c}n-n / c+r k \\ k\end{array}\right)-i$.

Все это надо еще разделить на $i$ !, поскольку мы добираем всего $i$ ребер, и начинать этот процесс можно с любого из этих $i$ ребер, то есть посчитали их ровно $i$ ! раз.

Предложение 1 доказано.

Докажем теперь (3). Нетрудно видеть, что

$$
\begin{aligned}
L(n) & =\frac{1}{N^{2}(n) \varepsilon^{2}} \sum_{\substack{P_{i}, P_{j} \in P G(n) \\
P_{i} \neq P_{j}}} \operatorname{cov}\left(I\left(P_{i}\right), I\left(P_{j}\right)\right)=\frac{M(n) R}{N^{2}(n) \varepsilon^{2}} \\
& =\frac{M(n)}{N^{2}(n) \varepsilon^{2}} \sum_{i=1}^{n /(k c)-1} R(i)\left(\left(\frac{D}{\left(\begin{array}{l}
n-1 \\
k-1
\end{array}\right)}\right)^{n /(k c)+i}-\left(\frac{D}{\left(\begin{array}{l}
n-1 \\
k-1
\end{array}\right)}\right)^{2 n /(k c)}\right) \\
& =\frac{1}{N(n) \varepsilon^{2}} \sum_{i=1}^{n /(k c)-1} R(i)\left(\left(\frac{D}{\left(\begin{array}{l}
n-1 \\
k-1
\end{array}\right)}\right)^{i}-\left(\frac{D}{\left(\begin{array}{l}
n-1 \\
k-1
\end{array}\right)}\right)^{n /(k c)}\right) \\
& \leqslant \frac{1}{N(n) \varepsilon^{2}} \sum_{i=1}^{n /(k c)-1} R(i)\left(\frac{D}{\left(\begin{array}{l}
n-1 \\
k-1
\end{array}\right)}\right)^{i} \leqslant(n /(k c)-1)_{i=1, \ldots, n /(k c)-1} \frac{R(i)}{N(n) \varepsilon^{2}}\left(\frac{D}{\left(\begin{array}{l}
n-1 \\
k-1
\end{array}\right)}\right)^{i}
\end{aligned}
$$




$$
\begin{aligned}
& =\left(\frac{n}{k c}-1\right)_{i=1, \ldots, n /(k c)-1} \frac{1}{N(n) \varepsilon^{2}}\left(\begin{array}{c}
n /(k c) \\
i
\end{array}\right) \frac{1}{i !} \prod_{r=1}^{i}\left(\left(\begin{array}{c}
n-n / c+r k \\
k
\end{array}\right)-i\right)\left(\frac{D}{\left(\begin{array}{l}
n-1 \\
k-1
\end{array}\right)}\right)^{i} \\
& =\left(\frac{n}{k c}-1\right) \max _{i=1, \ldots, n /(k c)-1} \frac{\left(\begin{array}{c}
n /(k c) \\
i
\end{array}\right)(1 / i !) \prod_{r=1}^{i}\left(\left(\begin{array}{c}
n-n / c+r k \\
k
\end{array}\right)-i\right)\left(D /\left(\begin{array}{c}
n-1 \\
k-1
\end{array}\right)\right)^{i}}{(1 /(n(k c)) !) \prod_{r=1}^{n /(k c)}\left(\begin{array}{c}
n-n / c+r k \\
k
\end{array}\right)\left(D /\left(\begin{array}{c}
n-1 \\
k-1
\end{array}\right)\right)^{n /(k c)} \varepsilon^{2}} \\
& \leqslant\left(\frac{n}{k c}-1\right) \max _{i=1, \ldots, n /(k c)-1} \frac{\left(\begin{array}{c}
n /(k c) \\
i
\end{array}\right)(1 / i !) \prod_{r=1}^{i}\left(\begin{array}{c}
n-n / c+r k \\
k
\end{array}\right)\left(D /\left(\begin{array}{c}
n-1 \\
k-1
\end{array}\right)\right)^{i}}{(1 /(n(k c))) \prod_{r=1}^{n /(k c)}\left(\begin{array}{c}
n-n / c+r k \\
k
\end{array}\right)\left(D /\left(\begin{array}{c}
n-1 \\
k-1
\end{array}\right)\right)^{n /(k c)} \varepsilon^{2}} \\
& \leqslant\left(\frac{n}{k c}-1\right) ! \max _{i=1, \ldots, n /(k c)-1} \frac{\left(\begin{array}{c}
n /(k c) \\
i
\end{array}\right)}{i !\left(D /\left(\begin{array}{c}
n-1 \\
k-1
\end{array}\right)\right)^{n /(k c)-i} \prod_{r=i+1}^{n /(k c)}\left(\begin{array}{c}
n-n / c+r k \\
k
\end{array}\right) \varepsilon^{2}} .
\end{aligned}
$$

Пусть

$$
B(i)=\frac{\left(\begin{array}{c}
n /(k c) \\
i
\end{array}\right)}{i !\left(D /\left(\begin{array}{c}
n-1 \\
k-1
\end{array}\right)\right)^{n /(k c)-i} \prod_{r=i+1}^{n /(k c)}\left(\begin{array}{c}
n-n / c+r k \\
k
\end{array}\right) \varepsilon^{2}}
$$

Тогда получаем, что

$$
L(n) \leqslant\left(\frac{n}{k c}+1\right) ! \max _{i=1, \ldots, n /(k c)-1} B(i) .
$$

Отсюда следует соотношение (3).

Предложение 2. Если выполнены условия теоремы 1, то В(i) монотонно возрастает no $i=1, \ldots, n /(k c)-1$.

Доказательство. Оценим снизу отношение $B(i) / B(i-1)$. Нетрудно видеть, что

$$
\begin{aligned}
\frac{B(i)}{B(i-1)} & =\frac{\left(\begin{array}{c}
n /(k c) \\
i
\end{array}\right)(i-1) !\left(D /\left(\begin{array}{c}
n-1 \\
k-1
\end{array}\right)\right)^{n /(k c)-i+1} \prod_{r=i}^{n /(k c)}\left(\begin{array}{c}
n-n / c+r k \\
k
\end{array}\right) \varepsilon^{2}}{i !\left(D /\left(\begin{array}{c}
n-1 \\
k-1
\end{array}\right)\right)^{n /(k c)-i} \prod_{r=i+1}^{n /(k c)}\left(\begin{array}{c}
n-n / c+r k \\
k
\end{array}\right) \varepsilon^{2}\left(\begin{array}{c}
n /(k c) \\
i-1
\end{array}\right)} \\
& =\frac{1}{i^{2}}\left(\frac{n}{k c}-i+1\right) D \frac{\left(\begin{array}{c}
n-n / c+i k \\
k
\end{array}\right)}{\left(\begin{array}{l}
n-1 \\
k-1
\end{array}\right)}=\frac{1}{c} \frac{(n /(k c)-i+1) D\left(\begin{array}{c}
n-n / c+i k \\
k
\end{array}\right)}{(n /(k c))\left(\begin{array}{c}
n \\
k
\end{array}\right) i^{2}} \\
& =\frac{1}{c} \frac{(n /(k c)-i+1) D}{n i^{2} / k c} \frac{(n-n / c+i k) !(n-k) !}{n !(n-n / c+(i-1) k) !} \\
& =\frac{1}{c} \frac{(n /(k c)-i+1) D}{n i^{2} /(k c)} \frac{\prod_{l=0}^{k-1}(n-n / c+i k-l)}{\prod_{l=0}^{k-1}(n-l)} \\
& \geqslant \frac{1}{c} \frac{(n /(k c)-i+1) D}{n i^{2} /(k c)} \frac{\prod_{l=0}^{k-1}(n-n / c-l)}{\prod_{l=0}^{k-1}(n-l)} .
\end{aligned}
$$

Поскольку $k=o(n)$ при $n \rightarrow \infty$, мы получаем, что при $n \rightarrow \infty$

$$
\begin{aligned}
\frac{1}{c} \frac{(n /(k c)-i+1) D}{n i^{2} /(k c)} \frac{\prod_{l=0}^{k-1}(n-n / c-l)}{\prod_{l=0}^{k-1}(n-l)} & \sim \frac{1}{c} \frac{(n /(k c)-i+1) D}{n i^{2} /(k c)}\left(\frac{n-n / c}{n}\right)^{k} \\
& =\frac{1}{c} \frac{(n /(k c)-i+1) D}{n i^{2} /(k c)}\left(1-\frac{1}{c}\right)^{k} .
\end{aligned}
$$


Отсюда видно, что при $D>c(n /(k c))^{3}\left(c /(c-1)^{k}\right.$ справедливо неравенство $B(i)>B(i-1)$ для любых $i=1, \ldots, n /(k c)-1$. Предложение 2 доказано.

Из предложения 2 и (4) получаем, что

$$
\begin{aligned}
L(n) & =\frac{\sum_{P_{i}, P_{j} \in P G(n)} \operatorname{cov}\left(I\left(P_{i}\right), I\left(P_{j}\right)\right)}{N^{2}(n) \varepsilon^{2}} \leqslant\left(\frac{n}{k c}+1\right) ! B\left(\frac{n}{k c}-1\right) \\
= & \left(\frac{n}{k c}+1\right) ! \frac{n /(k c)}{(n /(k c)-1) !\left(D /\left(\begin{array}{l}
n-1 \\
k-1
\end{array}\right)\right)\left(\begin{array}{l}
n \\
k
\end{array}\right) \varepsilon^{2}} \\
= & \frac{(n /(k c)(n /(k c)+1)}{D \varepsilon^{2}} \sim \frac{(n /(k c))^{2}}{D \varepsilon^{2}} \rightarrow 0
\end{aligned}
$$

при $n \rightarrow \infty$, так как

$$
D>c\left(\frac{n}{k c}\right)^{3}\left(\frac{c}{c-1}\right)^{k}
$$

Теорема 1 доказана.

Следствие 1. В условиях теоремь 1 для любого $\varepsilon>0$ при $n \rightarrow \infty$

$$
\mathbf{P}\left(\left|\frac{\sum_{P \in P G(n)} I(P)}{N(n)}-1\right|>\varepsilon\right) \rightarrow 0 .
$$

Замечание 2. Утверждение следствия 1 означает сходимость по вероятности к 1 отношения числа упаковок случайного гиперграфа $G(n, p)$ к его математическому ожиданию.

Замечание 3. В данном случае нельзя использовать закон больших чисел, потому что упаковки случайного гиперграфа - зависимые случайные величины.

\section{3. Асимптотика числа родившихся упаковок, содержащих фиксированное ребро $G(n)$}

Пусть $M(n)$ - число упаковок размера $n /(k c)$ полного гиперграфа $G(n)$, содержащих фиксированное ребро $A$ из множества ребер исходного полного гиперграфа $G(n)$. Это число совпадает с числом всех упаковок в $G(n)$ размера $n /(k c)-1$, все ребра которых не пересекают ребро $A$ (то есть не имеют с ним общих вершин). По аналогии с доказательством леммы 1 из [1] можно получить, что

$$
M(n)=\frac{\prod_{i=1}^{n /(k c)-1}\left(\begin{array}{c}
n-n / c+i k \\
k
\end{array}\right)}{(n /(k c)-1) !}=\frac{(n-k) !}{(k !)^{n /(k c)-1}(n(1-1 / c)) !(n /(k c)-1) !} .
$$

Пусть $P A G(n)$ - множество упаковок размера $n /(k c)-1$ в $G(n)$, каждое ребро которых не имеет общих вершин с ребром $A$.

Введем понятие числа упаковок случайного гиперграфа $G(n, p)$ размера $n /(k c)$, содержащих фиксированное ребро $A$ из множества ребер исходного полного гиперграфа $G(n)$. По определению, это случайная величина, равная сумме по всевозможным упаковкам $P$, содержащим ребро $A$, размера $n /(k c)$ полного гиперграфа $G(n)$ индикаторных случайных величин $I(P)$ :

$$
\sum_{P: P \in P G(n), P \ni A} I(P)=I(A) \sum_{P \in P A G(n)} I(P) .
$$


Подсчитаем ее математическое ожидание:

$$
\begin{aligned}
\mathbf{E}\left(\sum_{P: P \in P G(n), A \in P} I(P)\right) & =M(n)\left(\frac{D}{\left(\begin{array}{l}
n-1 \\
k-1
\end{array}\right)}\right)^{n /(k c)} \\
& =\frac{(n-k) !}{(k !)^{n /(k c)-1}(n(1-1 / c)) !(n /(k c)-1) !}\left(\frac{D}{\left(\begin{array}{l}
n-1 \\
k-1
\end{array}\right)}\right)^{n /(k c)} .
\end{aligned}
$$

Найдем математическое ожидание числа упаковок в $P A G(n)$ :

$$
\begin{aligned}
N(n) & =\mathbf{E}\left(\sum_{P \in P A G(n)} I(P)\right)=M(n)\left(\frac{D}{\left(\begin{array}{l}
n-1 \\
k-1
\end{array}\right)}\right)^{n /(k c)-1} \\
& =\frac{(n-k) !}{(k !)^{n /(k c)-1}(n(1-1 / c)) !(n /(k c)-1) !}\left(\frac{D}{\left(\begin{array}{l}
n-1 \\
k-1
\end{array}\right)}\right)^{n /(k c)-1} .
\end{aligned}
$$

Теорема 2. Пусть $G(n, p(n))$ - случайный гиперграф и при $n \rightarrow \infty$

$$
p(n)=\frac{D(n)}{\left(\begin{array}{c}
n-1 \\
k(n)-1
\end{array}\right)} \leqslant 1, \quad k(n)=o(n), \quad D(n)>c\left(\frac{n}{k(n) c}\right)^{3}\left(\frac{c}{c-1}\right)^{k(n)},
$$

где $k(n)$ - возрастающая функиия $n$. Тогда для любого $\varepsilon>0$ при $n \rightarrow \infty$

$\mathbf{P}\left(\left|\frac{1}{N(n)} \sum_{P: P \in P G(n), A \in P} I(P)-1\right|>\varepsilon \mid I(A)=1\right)<\frac{1}{\varepsilon^{2}}\left(\frac{1}{N(n)}+\frac{1}{(n / k(n))(c /(c-1))^{k(n)}}\right)$.

Доказательство. В качестве основного инструмента используется неравенство Чебышёва:

$$
\begin{aligned}
\mathbf{P}\left(\left|\frac{1}{N(n)} \sum_{P: P \in P G(n), A \in P} I(P)-1\right|>\varepsilon \mid I(A)=1\right) & =\mathbf{P}\left(\left|\frac{1}{N(n)} \sum_{P \in P A G(n)} I(P)-1\right|>\varepsilon\right) \\
& \leqslant \frac{1}{N^{2}(n) \varepsilon^{2}} \mathbf{D}\left(\sum_{P \in P A G(n)} I(P)\right),
\end{aligned}
$$

поскольку

$$
\sum_{P: P \in P G(n), A \in P} I(P)=I(A) \sum_{P \in P A G(n)} I(P)=\sum_{P \in P A G(n)} I(P)
$$

при $I(A)=1$, и $\sum_{P \in P A G(n)} I(P)$ не зависит от $I(A)$.

Далее мы рассматриваем упаковки $P$ только из множества $P A G(n)$. Ясно, что

$$
\mathbf{D}\left(\sum_{P \in P A G(n)} I(P)\right)=\sum_{P \in P A G(n)} \mathbf{D}(I(P))+2 \sum_{\substack{P_{i}, P_{j} \in P A G(n) \\ P_{i} \neq P_{j}}} \operatorname{cov}\left(I\left(P_{i}\right), I\left(P_{j}\right)\right) .
$$


Подсчитаем сумму дисперсий. Поскольку $I(P)-$ индикаторная случайная величина,

$$
\begin{aligned}
\mathbf{D}(I(P)) & =\left(\frac{D}{\left(\begin{array}{c}
n-1 \\
k-1
\end{array}\right)}\right)^{n /(k c)-1}\left(1-\left(\frac{D}{\left(\begin{array}{c}
n-1 \\
k-1
\end{array}\right)}\right)^{n /(k c)-1}\right) \Rightarrow \frac{1}{N^{2}(n) \varepsilon^{2}} \sum_{P \in P A G(n)} \mathbf{D}(I(P)) \\
& =\frac{1}{N^{2}(n) \varepsilon^{2}} M(n)\left(\frac{D}{\left(\begin{array}{l}
n-1 \\
k-1
\end{array}\right)}\right)^{n /(k c)-1}\left(1-\left(\frac{D}{\left(\begin{array}{l}
n-1 \\
k-1
\end{array}\right)}\right)^{n /(k c)-1}\right) \\
& =\frac{1}{N(n) \varepsilon^{2}}\left(1-\left(\frac{D}{\left(\begin{array}{c}
n-1 \\
k-1
\end{array}\right)}\right)^{n /(k c)-1}\right)<\frac{1}{N(n) \varepsilon^{2}} \rightarrow 0
\end{aligned}
$$

при $n \rightarrow \infty$, потому что

$$
N(n)=M(n)\left(\frac{D}{\left(\begin{array}{l}
n-1 \\
k-1
\end{array}\right)}\right)^{n /(k c)-1}
$$

Пусть

$$
L(n)=\frac{1}{N^{2}(n) \varepsilon^{2}} \sum_{\substack{P_{i}, P_{j} \in P A G(n) \\ P_{i} \neq P_{j}}} \operatorname{cov}\left(I\left(P_{i}\right), I\left(P_{j}\right)\right)
$$

Для доказательства теоремы 2 осталось показать, что при $n \rightarrow \infty$

$$
L(n) \rightarrow 0
$$

Если рассмотреть сумму всевозможных ковариаций некоторой фиксированной случайной упаковки $I(P), P \in P A G(n)$, и всех остальных случайных упаковок, то для каждой такой упаковки $I(P), P \in P A G(n)$, эта сумма будет одной и той же из соображений симметрии. Поэтому можно упростить выражение для суммы ковариаций следующим образом:

$$
R=\sum_{\substack{P_{i}, P_{j} \in P A G(n) \\ P_{i} \neq P_{j}}} \operatorname{cov}\left(I\left(P_{i}\right), I\left(P_{j}\right)\right)=M(n) \sum_{P_{j} \in P A G(n)} \operatorname{cov}\left(I(P), I\left(P_{j}\right)\right),
$$

где $P$ - любая фиксированная упаковка из $P A G(n)$.

Далее, выделим группы ковариаций в соответствии с числом общих ребер в упаковках $P$ и $P_{j}$ в $G(n)$. Для любого $i=1, \ldots, n /(k c)-2$ определим $R(i)$ как число упаковок в $G(n)$, имеющих в точности $i$ ребер, которых нет в упаковке $P$. Это означает, что оставшиеся $n /(k c)-i$ ребер каждой такой упаковки совпадают с какими-то ребрами упаковки $P$.

Если упаковка $P_{j}$ имеет в точности $n /(k c)-i$ ребер, которые есть в упаковке $P$ (то есть $i$ ее ребер нет в упаковке $P$ ), то

$\operatorname{cov}\left(P, P_{j}\right)=\mathbf{E}\left\{I(P) I\left(P_{j}\right)\right\}-\mathbf{E}\{I(P)\} \mathbf{E}\left\{I\left(P_{j}\right)\right\}=\left(\frac{D}{\left(\begin{array}{c}n-1 \\ k-1\end{array}\right)}\right)^{n(k c)-1+i}-\left(\frac{D}{\left(\begin{array}{l}n-1 \\ k-1\end{array}\right)}\right)^{2 n /(k c)-2}$.

Замечание 4. Если $i=n /(k c)-1$, то есть все ребра упаковок $P$ и $P_{j}$ различны, то случайные величины $I(P)$ и $I\left(P_{j}\right)$ независимы и $\operatorname{cov}\left(P, P_{j}\right)=0$.

Если $i=0, \operatorname{to} \operatorname{cov}\left(P, P_{j}\right)=\mathbf{D}(P)$, а сумма дисперсий уже оценена выше. 
Поэтому можно сгруппировать $R$ следующим образом:

$$
R=\sum_{i=1}^{n /(k c)-2} R(i)\left(\left(\frac{D}{\left(\begin{array}{l}
n-1 \\
k-1
\end{array}\right)}\right)^{n /(k c)-1+i}-\left(\frac{D}{\left(\begin{array}{l}
n-1 \\
k-1
\end{array}\right)}\right)^{2 n /(k c)-2}\right)
$$

Предложение 3. Для $i=1, \ldots, n /(k c)-2$

$$
R(i)=\left(\begin{array}{c}
n /(k c)-1 \\
i
\end{array}\right) \frac{1}{i !} \prod_{r=1}^{i}\left(\left(\begin{array}{c}
n-n / c+r k \\
k
\end{array}\right)-i\right) .
$$

Доказательство. Упаковка $P$ уже имеет $n /(k c)-i$ общих ребер с $P_{j}$. Надо добрать еще $i$ peбер.

Число способов выбрать из $n /(k c)-1$ ребер упаковки $P$ какие-то фиксированные $i$ ее ребер, по которым отличаются упаковка $P$ и другие упаковки $G(n)$ (то есть этих $i$ ребер в них нет, а все остальные ребра упаковки $P$ в них есть) равно $\left(\begin{array}{c}n /(k c)-1 \\ i\end{array}\right)$. Из симметрии полного гиперграфа следует, что при любом выборе этих $i$ ребер получим одно и то же число упаковок $G(n)$, которые отличаются от упаковки $P$ по этим $i$ ребрам.

Число способов выбрать $r$-е ребро упаковки $P_{j}$, которая уже имеет $n /(k c)-i$ общих ребер с $P$, есть $\left(\begin{array}{c}n-n / c+r k \\ k\end{array}\right)-i$, так как нельзя брать ребра, пересекающие ребро $A$ и эти $n /(k c)-i$ общих ребер с $P$.

Все это надо еще разделить на $i$ !, поскольку добираем всего $i$ ребер и начинать процесс добора ребер могли с любого из этих $i$ ребер, то есть посчитали их ровно $i$ ! раз. Предложение 3 доказано.

Докажем (5). Нетрудно видеть, что

$$
\begin{aligned}
L(n) & =\frac{1}{N^{2}(n) \varepsilon^{2}} \sum_{\substack{P_{i}, P_{j} \in P A G(n) \\
P_{i} \neq P_{j}}} \operatorname{cov}\left(I\left(P_{i}\right), I\left(P_{j}\right)\right)=\frac{M(n) R}{N^{2}(n) \varepsilon^{2}} \\
& =\frac{M(n)}{N^{2}(n) \varepsilon^{2}} \sum_{i=1}^{n /(k c)-2} R(i)\left(\left(\frac{D}{\left(\begin{array}{l}
n-1 \\
k-1
\end{array}\right)}\right)^{n /(k c)-1+i}-\left(\frac{D}{\left(\begin{array}{l}
n-1 \\
k-1
\end{array}\right)}\right)^{2 n /(k c)-2}\right) \\
& =\frac{1}{N(n) \varepsilon^{2}} \sum_{i=1}^{n /(k c)-2} R(i)\left(\left(\frac{D}{\left(\begin{array}{l}
n-1 \\
k-1
\end{array}\right)}\right)^{i}-\left(\frac{D}{\left(\begin{array}{l}
n-1 \\
k-1
\end{array}\right)}\right)^{n /(k c)-1}\right)^{n /(k c)-2} \sum_{i=1}^{n(n) \varepsilon^{2}} R(i)\left(\frac{D}{\left(\begin{array}{l}
n-1 \\
k-1
\end{array}\right)}\right)^{i} \\
& \leqslant\left(\frac{n}{k c}-2\right) \underset{\substack{i=1, \ldots, n /(k c)-2 \\
\max _{i}}}{N(n) \varepsilon^{2}} R(i)\left(\frac{D}{\left(\begin{array}{l}
n-1 \\
k-1
\end{array}\right)}\right)^{i} .
\end{aligned}
$$

Продолжая эту цепочку неравенств с учетом предложения 3, получаем, что

$$
\begin{aligned}
L(n)= & \left(\frac{n}{k c}-2\right) \frac{1}{N(n) \varepsilon^{2}}\left(\begin{array}{c}
n /(k c)-1 \\
i
\end{array}\right) \frac{1}{i !} \prod_{r=1}^{i}\left(\left(\begin{array}{c}
n-n / c+r k \\
k
\end{array}\right)-i\right)\left(\frac{D}{\left(\begin{array}{l}
n-1 \\
k-1
\end{array}\right)}\right)^{i} \\
& \times \max _{i=1, \ldots, n /(k c)-2} \\
= & \left.\frac{n}{k c}-2\right) \\
& \times \max _{i=1, \ldots, n /(k c)-2} \frac{\left(\begin{array}{c}
n /(k c)-1 \\
i
\end{array}\right)(1 / i !) \prod_{r=1}^{i}\left(\left(\begin{array}{c}
n-n / c+r k \\
k
\end{array}\right)-i\right)\left(D /\left(\begin{array}{l}
n-1 \\
k-1
\end{array}\right)\right)^{i}}{1 /((n /(k c)-1) !)\left(\prod_{r=1}^{n /(k c)-1}\left(\begin{array}{c}
n-n / c+r k \\
k
\end{array}\right)\right)\left(D /\left(\begin{array}{l}
n-1 \\
k-1
\end{array}\right)\right)^{n /(k c)-1} \varepsilon^{2}}
\end{aligned}
$$




$$
\begin{aligned}
& =\left(\frac{n}{k c}-2\right) \\
& \times \max _{i=1, \ldots, n /(k c)-2} \frac{i}{(1 /(n /(k c)-1) !)\left(\prod_{r=1}^{n /(k c)-1}\left(\begin{array}{c}
n-n / c+r k \\
k
\end{array}\right)\right)\left(D /\left(\begin{array}{c}
n-1 \\
k-1
\end{array}\right)\right)^{n /(k c)-1} \varepsilon^{2}} \\
& \leqslant\left(\frac{n}{k c}\right) ! \max _{i=1, \ldots, n /(k c)-2} \frac{\left(\begin{array}{c}
n /(k c)-1 \\
i
\end{array}\right)}{i !\left(D /\left(\begin{array}{c}
n-1 \\
k-1
\end{array}\right)\right)^{n /(k c)-1-i} \prod_{r=i+1}^{n /(k c)-1}\left(\begin{array}{c}
n-n / c+r k \\
k
\end{array}\right) \varepsilon^{2}} .
\end{aligned}
$$

Положим

$$
B(i)=\frac{\left(\begin{array}{c}
n /(k c)-1 \\
i
\end{array}\right)}{i !\left(D /\left(\begin{array}{c}
n-1 \\
k-1
\end{array}\right)\right)^{n /(k c)-1-i} \prod_{r=i+1}^{n /(k c)-1}\left(\begin{array}{c}
n-n / c+r k \\
k
\end{array}\right) \varepsilon^{2}} .
$$

Тогда

$$
L(n) \leqslant\left(\frac{n}{k c}\right) ! \max _{i=1, \ldots, n /(k c)-2} B(i) .
$$

Предложение 4. В условиях теоремы 2 функция $B(i)$ монотонно возрастает с ростом $i=1, \ldots, n /(k c)-2$.

Доказательство. Оценим снизу отношение $B(i) / B(i-1)$. Нетрудно видеть, что

$$
\begin{aligned}
\frac{B(i)}{B(i-1)} & =\frac{\left(\begin{array}{c}
n /(k c-1) \\
i
\end{array}\right)(i-1) !\left(D /\left(\begin{array}{l}
n-1 \\
k-1
\end{array}\right)\right)^{n /(k c)-1-i+1} \prod_{r=i}^{n /(k c)-1}\left(\begin{array}{c}
n-n / c+r k \\
k
\end{array}\right) \varepsilon^{2}}{i !\left(D /\left(\begin{array}{c}
n-1 \\
k-1
\end{array}\right)\right)^{n /(k c)-1-i} \prod_{r=i+1}^{n /(k c)-1}\left(\begin{array}{c}
n-n / c+r k \\
k
\end{array}\right) \varepsilon^{2}\left(\begin{array}{c}
n /(k c)-1 \\
i-1
\end{array}\right)} \\
& =\frac{(n /(k c)-i)\left(D /\left(\begin{array}{c}
n-1 \\
k-1
\end{array}\right)\right)\left(\begin{array}{c}
n-n / c+i k \\
k
\end{array}\right)}{i^{2}}=\frac{1}{c} \frac{(n /(k c)-i) D\left(\begin{array}{c}
n-n / c+i k \\
k
\end{array}\right)}{\left(n i^{2} /(k c)\right)\left(\begin{array}{l}
n \\
k
\end{array}\right)} \\
& =\frac{1}{c} \frac{(n /(k c)-i) D}{n i^{2} /(k c)} \frac{(n-n / c+i k) !(n-k) !}{n !(n-n / c+(i-1) k) !} \\
& =\frac{1}{c} \frac{(n /(k c)-i) D}{n i^{2} /(k c)} \frac{\prod_{l=0}^{k-1}(n-n / c+i k-l)}{\prod_{l=0}^{k-1}(n-l)} q \\
& \geqslant \frac{1}{c} \frac{(n /(k c)-i) D}{n i^{2} /(k c)} \frac{\prod_{l=0}^{k-1}(n-n / c-l)}{\prod_{l=0}^{k-1}(n-l)} .
\end{aligned}
$$

Учитывая, что $k=o(n)$ при $n \rightarrow \infty$, находим, что последний член в полученной цепочке неравенств при $n \rightarrow \infty$ эквивалентен

$$
\frac{1}{c} \frac{(n /(k c)-i) D}{n i^{2} /(k c)}\left(\frac{n-n / c}{n}\right)^{k}=\frac{1}{c} \frac{(n /(k c)-i) D}{n i^{2} /(k c)}\left(1-\frac{1}{c}\right)^{k} .
$$

Отсюда видно, что при

$$
D>c\left(\frac{n}{k c}\right)^{3}\left(\frac{c}{c-1}\right)^{k}
$$

выполняется неравенство $B(i)>B(i-1)$ для любых $i=1, \ldots, n / k c-2$. Предложение 4 доказано. 
Из предложения 4 и (6) получаем, что

$$
\begin{aligned}
L(n) & =\frac{\sum_{P_{i}, P_{j} \in P A G(n)} \operatorname{cov}\left(I\left(P_{i}\right), I\left(P_{j}\right)\right)}{P_{i} \neq P_{j}}\left(\frac{n}{k c}\right) ! B\left(\frac{n}{k c}-2\right) \\
= & \left(\frac{n}{k c}\right) ! \frac{n /(k c)-1}{(n /(k c)-2) !\left(D /\left(\begin{array}{l}
n-1 \\
k-1
\end{array}\right)\right)\left(\begin{array}{l}
n \\
k
\end{array}\right) \varepsilon^{2}}=\frac{(n /(k c))(n /(k c)-1)}{D \varepsilon^{2}} .
\end{aligned}
$$

При $n \rightarrow \infty$ последний член цепочки эквивалентен $(n /(k c))^{2} /\left(D \varepsilon^{2}\right)$ и стремится к нулю, поскольку $D>c(n /(k c))^{3}(c /(c-1))^{k}$. Теорема 2 доказана.

Следствие 2. В условиях теоремы 2 для любого $\varepsilon>0$ при $n \rightarrow \infty$

$$
\mathbf{P}\left(\left|\frac{1}{N} \sum_{P \in P G(n), A \in P} I(P)-1\right|>\varepsilon \mid I(A)=1\right) \rightarrow 0 .
$$

Замечание 5. Утверждение следствия 2 означает сходимость по вероятности к 1 отношения числа упаковок случайного гиперграфа $G(n, p)$, содержащих ребро $A$ из $G(n)$, и некоторой случайной величины, похожей на его математическое ожидание.

Замечание 6. В данном случае нельзя использовать закон больших чисел, потому что упаковки случайного гиперграфа - зависимые случайные величины.

\section{4. Оценка сверху хроматического индекса случайного гиперграфа}

Теорема 3. Пусть $G(n, p(n))$ - случайный гиперграф,

$$
p(n)=\frac{D(n)}{\left(\begin{array}{c}
n-1 \\
k(n)-1
\end{array}\right)} \leqslant 1, \quad D(n)>c\left(\frac{n}{k(n) c}\right)^{3}\left(\frac{c}{c-1}\right)^{k(n)},
$$

где $k(n)$ - возрастающиая функция от $n$ и $k(n)=$ o(n) при $n \rightarrow \infty$. Тогда при $n \rightarrow \infty$ для любого $\varepsilon>0$

$$
\mathbf{P}(\chi(G(n, p(n))) \leqslant[1+\varepsilon+c \ln (k(n))] D(n)) \rightarrow 1 .
$$

Доказательство. Введем следующее вероятностное пространство. Пусть $\Omega_{0}-$ множество векторов из 0 и 1 длины $\left(\begin{array}{l}n \\ k\end{array}\right)$, каждый из которых соответствует конкретному подмно-

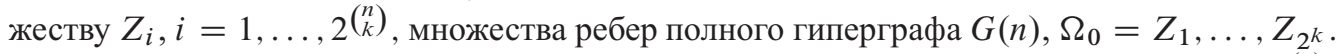
Определим независимые в совокупности случайные величины $\xi_{0}, \xi_{i}^{j}, i=1, \ldots, 2\left(\begin{array}{c}n \\ k\end{array}\right)$, $1 \leqslant j \leqslant c D \ln (k)$, следующим образом. Определим дискретную случайную величину $\xi_{0}$, которая описывает процесс равномерного и независимого выбора ребер $G(n)$ :

$$
\begin{array}{r}
\mathbf{P}\left(\xi_{0}=\left(e_{1}, \ldots, e_{\left(\begin{array}{c}
n \\
k
\end{array}\right)}\right): e_{i_{1}}=1, e_{i_{2}}=1, e_{i_{r}}=1, \text { остальные } e_{j}=0\right)=\left(\begin{array}{l}
n \\
r
\end{array}\right) p^{r}(1-p)^{n-r}, \\
0 \leqslant r \leqslant n, \quad r \in \mathbf{Z} .
\end{array}
$$


Далее, для каждого набора ребер, соответствующего $Z_{i}$, определяем множество $\Omega_{i}$, $i=1, \ldots, 2^{k}$, всех упаковок размера $n /(k c)$, которые можно построить на ребрах $Z_{i}$ (какие-то $\Omega_{i}=\varnothing$ ). Эти множества необходимы для описания процесса случайного и независимого выбора упаковок в случайном гиперграфе $G(n, p)$.

Далее выберем $c D \ln (k)$ равномерно распределенных упаковок случайного гиперграфа $G(n, p)$. Для этого определим случайные величины, введенные ранее, следующим образом: для всех $i \in\left\{1, \ldots, 2\left(\begin{array}{c}n \\ k\end{array}\right)\right\}, j \in\{1, \ldots, c D \ln (k)\}$ положим

$$
\mathbf{P}\left(\xi_{i}^{j}=P\right)=\frac{1}{\left|\Omega_{i}\right|}, \quad P \in \Omega_{i}, \quad|P|=\frac{n}{k c} .
$$

Если $\Omega_{i}=\varnothing$, то $\mathbf{P}\left(\xi_{i}^{j}=0\right)=1$ есть специально выделенный элемент для того, чтобы показать невозможность выбора упаковки размера $n /(k c)$ на $Z_{i}$. Если $\Omega_{i} \neq \varnothing$, то $\mathbf{P}\left(\xi_{i}^{j}=0\right)=0$.

Доказательство того, что существует вероятностное пространство $(\Omega, \mathscr{F}, \mathbf{P})$, на котором определены данные случайные величины, приведено на стр. 314-323 в [3].

На этом вероятностном пространстве определим случайные величины

$$
\eta_{j}=\sum_{i=1}^{2\left(\begin{array}{c}
n \\
k
\end{array}\right)} I\left(\xi_{0}=Z_{i}\right) \xi_{i}^{j}, \quad j=1, \ldots, \leqslant c D \ln (k) .
$$

Эти случайные величины описывают процесс равномерного выбора упаковки из $\Omega_{i}$.

Введем дополнительно случайные величины - индикаторы событий $I\left(\xi_{0}=Z_{i}\right)=1$, происходящих с вероятностью $\mathbf{P}\left(\xi_{0}=Z_{i}\right)$, где $\xi_{0}$ соответствует последовательности независимых испытаний Бернулли, как отмечалось ранее.

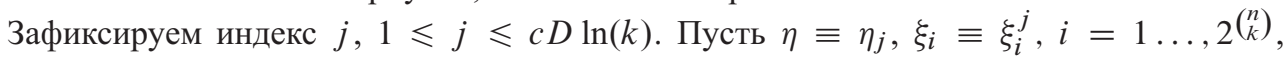
$A \in E(G(n))$ - фиксированное ребро полного гиперграфа на $n$ вершинах. Формальная запись того, что это ребро принадлежит случайной упаковке $\eta$, если оно родилось, то есть принадлежит случайному гиперграфу $G(n, p)$, такова:

$$
\begin{aligned}
\text { Событие }(A \text { родилось }) & =\left(\bigcup_{i: Z_{i} \ni A}\left(I\left(\xi_{0}=Z_{i}\right)=1\right)\right), \\
\text { Событие }(A \in \eta \& A \text { родилось }) & =\left(\bigcup_{i: A \in Z_{i}}\left(\left(\xi_{0}=Z_{i}\right) \&\left(\bigcup_{P: \Omega_{i} \ni P \ni A}\left(\xi_{i}=P\right)\right)\right)\right) .
\end{aligned}
$$

Оценим вероятность последнего события с учетом независимости в совокупности случайных величин $\xi_{0}, \xi_{i}, i \in\left\{1, \ldots, 2\left(\begin{array}{c}n \\ k\end{array}\right)\right\}$ :

$$
\begin{aligned}
\mathbf{P}(A \in \eta \& A \text { родилось }) & =\mathbf{P}\left(\bigcup_{i: A \in Z_{i}}\left(\left(\xi_{0}=Z_{i}\right) \&\left(\bigcup_{P: \Omega_{i} \ni P \ni A}\left(\xi_{i}=P\right)\right)\right)\right) \\
& =\sum_{i: Z_{i} \ni A} \sum_{P: \Omega_{i} \ni P \ni A} \mathbf{P}\left(\xi_{i}=P\right) \mathbf{P}\left(\xi_{0}=Z_{i}\right) \\
& =\sum_{i: Z_{i} \ni A} \sum_{P: \Omega_{i} \ni P \ni A} \frac{1}{\left|\Omega_{i}\right|} \mathbf{P}\left(\xi_{0}=Z_{i}\right) .
\end{aligned}
$$


Далее используем теоремы 1 и 2 для того, чтобы получить асимптотику этой вероятности. В теореме 1 говорится о том, что число родившихся упаковок отклоняется от своего математического ожидания не более, чем в $\left(1+\varepsilon_{1}\right)$ раз на мере, стремящейся к 1 при $n \rightarrow \infty$. В теореме 2 говорится о том, что число родившихся упаковок, содержащих фиксированное ребро $A$ полного гиперграфа $G(n)$, отклоняется от своего математического ожидания не более, чем в $\left(1+\varepsilon_{2}\right)$ раз на мере, стремящейся к 1 при $n \rightarrow \infty$ при условии, что ребро $A$ родилось. Из доказательства теорем 1 и 2 следует, что можно выбрать $\varepsilon_{1}(n)$ и $\varepsilon_{2}(n)$ как функции от $n$, стремящиеся к 0 при $n \rightarrow \infty$. Следовательно, можно выделить упаковку с вероятностью, не меньшей $1 /(c D)$ при $n \rightarrow \infty$. При $n \rightarrow \infty$

$\mathbf{P}(A \in \eta \& A$ родилось $)$

$$
\begin{aligned}
& \frac{\frac{(n-k) !}{(k !)^{n /(k c)-1}(n(1-1 / c)) !(n /(k c)-1) !}\left(\frac{D}{\left(\begin{array}{c}
n-1 \\
k-1
\end{array}\right)}\right)^{n /(k c)-1}}{\frac{n !}{(k !)^{n /(k c)}(n(1-1 / c)) !(n /(k c)) !}\left(\frac{D}{\left(\begin{array}{c}
n-1 \\
k-1
\end{array}\right)}\right)^{n /(k c)}} \mathbf{P}(A \text { родилось }) \\
= & \frac{1}{c D} \mathbf{P}(A \text { родилось }) \Rightarrow \mathbf{P}(A \in \eta \mid A \text { родилось }) \gtrsim \frac{1}{c D} .
\end{aligned}
$$

Таким образом, мы получили следующее утверждение.

Предложение 5. Для любого ребра $A \in E(G(n))$ nри $n \rightarrow \infty$

$$
\mathbf{P}(A \in \eta \mid \text { А родилось }) \gtrsim \frac{1}{c D} .
$$

Предложение 6. Для любого ребра $A \in E(G(n))$ nри $n \rightarrow \infty$

$$
\mathbf{P}(A \notin \eta \mid \text { А родилось }) \gtrsim 1-\frac{1}{c D} .
$$

Доказательство. Оценим снизу

$$
\mathbf{P}(A \notin \eta \& A \text { родилось })=\mathbf{P}\left(\bigcup_{i: A \in Z_{i}}\left(\left(\xi_{0}=Z_{i}\right) \&\left(\bigcup_{P: \Omega_{i} \ni P, A \notin P}\left(\xi_{i}=P\right)\right)\right)\right) \text {. }
$$

В силу независимости случайных величин $\xi_{0}, \xi_{i}, i \in\left\{1, \ldots, 2\left(\begin{array}{l}n \\ k\end{array}\right)\right\}$, для этой вероятности получаем оценку снизу

$$
\begin{aligned}
\sum_{i: Z_{i} \ni A} \sum_{P: \Omega_{i} \ni P, A \notin P} \mathbf{P}\left(\xi_{i}=P\right) \mathbf{P}\left(\xi_{0}=Z_{i}\right) & =\sum_{i: Z_{i} \ni A} \sum_{P: \Omega_{i} \ni P, A \notin P} \frac{1}{\left|\Omega_{i}\right|} \mathbf{P}\left(\xi_{0}=Z_{i}\right) \\
& =\sum_{i: Z_{i} \ni A} \mathbf{P}\left(\xi_{0}=Z_{i}\right)\left(1-\sum_{P: \Omega_{i} \ni P \ni A} \frac{1}{\left|\Omega_{i}\right|}\right) .
\end{aligned}
$$


Проведя те же рассуждения, что и при доказательстве предложения 5, получим, что

$\mathbf{P}(A \notin \eta \& A$ родилось $)$

$$
\begin{aligned}
& \gtrsim\left(1-\frac{\frac{(n-k) !}{(k !)^{n /(k c)-1}(n(1-1 / c)) !(n /(k c)-1) !}\left(\frac{D}{\left(\begin{array}{c}
n-1 \\
k-1
\end{array}\right)}\right)^{n /(k c)-1}}{\frac{n !}{(k !)^{n /(k c)}(n(1-1 / c)) !(n /(k c)) !}\left(\frac{D}{\left(\begin{array}{c}
n-1 \\
k-1
\end{array}\right)}\right)^{n /(k c)}}\right) \mathbf{P}(A \text { родилось }) \\
& =\left(1-\frac{1}{c D}\right) \mathbf{P}(A \text { родилось }) \Rightarrow \mathbf{P}(A \notin \eta \mid A \text { родилось }) \gtrsim\left(1-\frac{1}{c D}\right) .
\end{aligned}
$$

Предложение 6 доказано.

Предложение 7. Для любого ребра $A \in E(G(n))$ nри $n \rightarrow \infty$

$$
\mathbf{P}(A \notin \eta \mid A \text { родилось }) \sim 1-\frac{1}{c D} .
$$

Доказательство. По формуле полной вероятности

$$
\begin{aligned}
\mathbf{P}(A \text { родилось }) & =\mathbf{P}(A \notin \eta \& A \text { родилось })+\mathbf{P}(A \in \eta \& A \text { родилось }) \\
& \Rightarrow \mathbf{P}(A \notin \eta \mid A \text { родилось })=\mathbf{P}(A \text { родилось })-\mathbf{P}(A \in \eta \& A \text { родилось }) \\
& \lesssim \mathbf{P}(A \text { родилось })-\frac{1}{c D} \mathbf{P}(A \text { родилось })=\left(1-\frac{1}{c D}\right) \mathbf{P}(A \text { родилось }) .
\end{aligned}
$$

Здесь использовалось предложение 5. Из предложения 6 следует, что нижняя асимптотическая оценка будет такой же. Предложение 7 доказано.

Из предложения 7 следует, что

$$
\mathbf{P}(A \in \eta \mid A \text { родилось }) \sim \frac{1}{c D} .
$$

Отметим полезное свойство, которое пригодится в дальнейшем: при $n \rightarrow \infty$

$$
\mathbf{P}\left(A \in \xi_{i}\right)=\sum_{P: \Omega_{i} \ni P \ni A} P\left(\xi_{i}=P\right) \sim \frac{1}{c D} .
$$

Это верно для почти всех $i \in\left\{1, \ldots, 2\left(\begin{array}{l}n \\ k\end{array}\right)\right\}$ в силу ранее доказанного.

Теперь проделаем похожие вычисления, но не для одной случайной упаковки $\eta$, а для $c D \ln (k)$ упаковок $\eta_{j}, j \in\{1, \ldots, c D \ln (k)\}$.

Предложение 8. Для любого ребра $A \in E(G(n))$ nри $n \rightarrow \infty$

$$
\mathbf{P}\left(A \notin \eta_{j}, j \in\{1, \ldots, c D \ln (k)\} \mid A \text { родилось }\right) \sim \frac{1}{k} .
$$

Доказательство. Найдем асимптотику при $n \rightarrow \infty$ для вероятности

$$
\begin{aligned}
\mathbf{P}\left(A \notin \eta_{j}, j \in\{1, \ldots, c D \ln (k)\} \& A \text { родилось }\right) \\
=\mathbf{P}\left(\bigcup_{i: A \in Z_{i}}\left(\left(\xi_{0}=Z_{i}\right) \&\left(\bigcap_{j \in\{1, \ldots, c D \ln (k)\} P: \Omega_{i} \ni P, A \notin P}\left(\xi_{i}^{j}=P\right)\right)\right)\right) .
\end{aligned}
$$


Учитывая независимость в совокупности случайных величин $\xi_{0}, \xi_{i}^{j}, i \in\left\{1, \ldots, 2\left(\begin{array}{c}n \\ k\end{array}\right)\right\}$, $j \in\{1, \ldots, c D \ln (k)\}$, и одинаковую распределенность $\xi_{i}^{j}, i \in\left\{1, \ldots, 2\left(\begin{array}{l}n \\ k\end{array}\right)\right\}$, для $j \in\{1, \ldots, c D \ln (k)\})$, и продолжая цепочку равенств, получаем, что

$$
\begin{aligned}
& \mathbf{P}\left(A \notin \eta_{j}, j \in\{1, \ldots, c D \ln (k)\} \& A \text { родилось }\right) \\
& =\sum_{i: Z_{i} \ni A}\left(\sum_{P: \Omega_{i} \ni P, A \notin P} \mathbf{P}\left(\xi_{i}=P\right)\right)^{c D \ln (k)} \mathbf{P}\left(\xi_{0}=Z_{i}\right) \\
& =\sum_{i: Z_{i} \ni A}\left(\sum_{P: \Omega_{i} \ni P, A \notin P} \frac{1}{\left|\Omega_{i}\right|}\right)^{c D \ln (k)} \mathbf{P}\left(\xi_{0}=Z_{i}\right) \\
& =\sum_{i: Z_{i} \ni A} \mathbf{P}\left(\xi_{0}=Z_{i}\right)\left(1-\sum_{P: \Omega_{i} \ni P \ni A} \frac{1}{\left|\Omega_{i}\right|}\right)^{c D \ln (k)} \sim \sum_{i: Z_{i} \ni A} \mathbf{P}\left(\xi_{0}=Z_{i}\right)\left(1-\frac{1}{c D}\right)^{c D \ln (k)} \\
& =\mathbf{P}(A \text { родилось }) e^{\ln (1-1 /(c D)) c D \ln (k)} \sim \mathbf{P}(A \text { родилось }) e^{-1 /(c D) c D \ln (k)}=\frac{1}{k} \mathbf{P}(A \text { родилось }) .
\end{aligned}
$$

Предложение 8 доказано.

Замечание 7. В предпоследнем предельном переходе предполагалось, что $\varepsilon_{1}$ и $\varepsilon_{2}$ из теорем 1 и 2 можно выбрать как функции от $n$, например, порядка $\left.\left(k^{2} / n\right)(c-1) / c\right)^{k}$.

Таким образом, получена асимптотика вероятности того, что фиксированное ребро из полного гиперграфа $G(n)$ не входит ни в одну из выбранных $c D \ln (k)$ случайных упаковок. Определим случайный гиперграф $G^{\prime}$ как объединение этих случайных упаковок, то есть

$$
G^{\prime}=\eta_{1} \cup \eta_{2} \cup \ldots \cup \eta_{c D} \ln (k) .
$$

Тогда его хроматический индекс удовлетворяет неравенству

$$
\chi\left(G^{\prime}\right) \leqslant c D \ln (k) .
$$

Если определить $\widetilde{G}=G(n, p) \backslash G^{\prime}$ как гиперграф, который остался от $G(n, p)$ после исключения из множества его ребер всех ребер гиперграфа $G^{\prime}$, то, используя оценку (1), для его хроматического индекса можно получить оценку

$$
\chi(\widetilde{G}) \leqslant k\left(\max _{v \in V(\widetilde{G})} \operatorname{deg} \widetilde{G} v-1\right)+1,
$$

где $\operatorname{deg}_{\widetilde{G}} v-$ степень вершины $v$ в гиперграфе $\widetilde{G}$. Осталось оценить

$$
\operatorname{deg}_{\widetilde{G}} v=\sum_{A: A \ni v} I\left(A \text { родилось } \& A \notin \eta_{j}, 1 \leqslant j \leqslant c D \ln (k)\right) \text {. }
$$

Здесь $I(X)$ - индикатор события $X$, то есть $I(X)=1$ с вероятностью $\mathbf{P}(X), I(X)=0$ с вероятностью $1-\mathbf{P}(X)$. Подсчитаем математическое ожидание этой случайной величины:

$$
\mathbf{E}\left(\operatorname{deg}_{\widetilde{G}} v\right)=\left(\begin{array}{l}
n-1 \\
k-1
\end{array}\right) \mathbf{P}\left(A \text { родилось } \& A \notin \eta_{j}, 1 \leqslant j \leqslant c D \ln (k)\right) \text {. }
$$


Используя предложение 8 для получения асимптотики этой вероятности, находим, что при $n \rightarrow \infty$

$$
\mathbf{E}\left(\operatorname{deg}_{\widetilde{G}} v\right) \sim\left(\begin{array}{l}
n-1 \\
k-1
\end{array}\right) \frac{D}{k\left(\begin{array}{l}
n-1 \\
k-1
\end{array}\right)}=\frac{D}{k} .
$$

Далее надо показать, что $\operatorname{deg}_{\widetilde{G}} v$ отклоняется асимптотически от своего математического ожидания с небольшой вероятностью.

Предложение 9. При $n \rightarrow \infty$ для любого $\varepsilon>0$

$$
\mathbf{P}\left(\left|\frac{\operatorname{deg}_{\widetilde{G}} v}{D / k}-1\right|>\varepsilon\right) \rightarrow 0
$$

Доказательство. Используем, как и ранее, неравенство Чебышёва для оценки этой вероятности. Рассмотрим события

$$
\begin{aligned}
& A=\left(\text { ребро } A \text { родилось } \& A \notin \eta_{j}, 1 \leqslant j \leqslant c D \ln (k)\right), \\
& B=\left(\text { ребро } B \text { родилось } \& B \notin \eta_{j}, 1 \leqslant j \leqslant c D \ln (k)\right) .
\end{aligned}
$$

Нетрудно видеть, что

$$
\mathbf{P}\left(\left|\frac{\operatorname{deg}_{\widetilde{G}} v}{D / k}-1\right|>\varepsilon\right) \leqslant \frac{\sum_{A: A \ni v} \mathbf{D}(I(A))+\sum_{A \neq B: A \ni v, B \ni v} \operatorname{cov}(I(A), I(B))}{\varepsilon^{2} D^{2} / k^{2}}
$$

и при $n \rightarrow \infty$

$$
\begin{aligned}
\mathbf{D}(I(A)) & =\mathbf{P}(A)(1-\mathbf{P}(A)) \leqslant \mathbf{P}(A)=\frac{D}{\left(\begin{array}{l}
n-1 \\
k-1
\end{array}\right)} \Rightarrow \sum_{A: A \ni v} \mathbf{D}(I(A)) \leqslant\left(\begin{array}{l}
n-1 \\
k-1
\end{array}\right) \frac{D}{\left(\begin{array}{l}
n-1 \\
k-1
\end{array}\right)}=D \\
& \Rightarrow \frac{\sum_{A: A \ni v} \mathbf{D}(I(A))}{\varepsilon^{2} D^{2} / k^{2}} \rightarrow 0,
\end{aligned}
$$

поскольку по условию

$$
D>c\left(\frac{n}{k c}\right)^{3}\left(\frac{c}{c-1}\right)^{k}
$$

и $k=o(n)$ при $n \rightarrow \infty$. Поэтому осталось оценить $\sum_{A \neq B: A \ni v, B \ni v} \operatorname{cov}(I(A), I(B))$. Нетрудно видеть, что

$$
\operatorname{cov}(I(A), I(B))=\mathbf{P}(A \& B)-\mathbf{P}(A) \mathbf{P}(B)
$$

И

$$
\mathbf{P}(A \& B)=\mathbf{P}\left(\bigcup_{i: A \in Z_{i}, B \in Z_{i}}\left(\left(\xi_{0}=Z_{i}\right) \&\left(\bigcap_{1 \leqslant j \leqslant c D \ln (k) P: \Omega_{i} \ni P, A \notin P, B \notin P}\left(\xi_{i}^{j}=P\right)\right)\right)\right) .
$$

Учитывая независимость в совокупности случайных величин $\xi_{0}, \xi_{i}^{j}, i \in\left\{1, \ldots, 2\left(\begin{array}{c}n \\ k\end{array}\right)\right\}$, $1 \leqslant j \leqslant c D \ln (k)$, и одинаковую распределенность величин $\xi_{i}^{j}, i \in\left\{1, \ldots, 2\left(\begin{array}{c}n \\ k\end{array}\right)\right\}$, 
$1 \leqslant j \leqslant c D \ln (k)$, и продолжая оценивание, находим, что при $n \rightarrow \infty$

$$
\begin{aligned}
\mathbf{P}(A \& B)= & \sum_{i: Z_{i} \ni A, Z_{i} \ni B}\left(\sum_{P: \Omega_{i} \ni P, A \notin P, B \notin P} \mathbf{P}\left(\xi_{i}=P\right)\right)^{c D \ln (k)} \mathbf{P}\left(\xi_{0}=Z_{i}\right) \\
= & \sum_{i: Z_{i} \ni A, Z_{i} \ni B}\left(\sum_{P: \Omega_{i} \ni P, A \notin P, B \notin P} \frac{1}{\left|\Omega_{i}\right|}\right)^{c D \ln (k)} \mathbf{P}\left(\xi_{0}=Z_{i}\right) \\
= & \sum_{i: Z_{i} \ni A, Z_{i} \ni B} \mathbf{P}\left(\xi_{0}=Z_{i}\right)\left(1-\sum_{P: \Omega_{i} \ni P \ni A} \frac{2}{\left|\Omega_{i}\right|}\right)^{c D \ln (k)} \\
& \sum_{i: Z_{i} \ni A, Z_{i} \ni \text { э }} \mathbf{P}\left(\xi_{0}=Z_{i}\right)\left(1-\frac{2}{c D}\right)^{c D \ln (k)} \\
= & \mathbf{P}(A, B \text { родились }) e^{\ln (1-2 /(c D)) c D \ln (k)} \\
\sim & \mathbf{P}(A, B \text { родились }) e^{-2 /(c D) c D \ln (k)} \\
= & \frac{1}{k^{2}} \mathbf{P}(A \text { родилось }) \mathbf{P}(B \text { родилось }) .
\end{aligned}
$$

Асимптотика $\mathbf{P}(A), \mathbf{P}(B)$ была найдена ранее (см. предложение 8). Теперь можно оценить ковариацию: если $n \rightarrow \infty$, то

$$
\begin{aligned}
\operatorname{cov}(I(A), I(B))= & \mathbf{P}(A \& B)-\mathbf{P}(A) \mathbf{P}(B) \\
= & \mathbf{P}(A \text { родилось }) \mathbf{P}(B \text { родилось })\left(\frac{1}{k^{2}}+o\left(\frac{1}{k^{2}}\right)\right) \\
& \quad-\mathbf{P}(A \text { родилось }) \mathbf{P}(B \text { родилось })\left(\frac{1}{k^{2}}+o\left(\frac{1}{k^{2}}\right)\right) \\
= & o\left(\frac{1}{k^{2}}\right)\left(\frac{D}{\left(\begin{array}{c}
n-1 \\
k-1
\end{array}\right)}\right)^{2} \Rightarrow \frac{\sum_{A \neq B: A \ni v, B \ni v} \operatorname{cov}(I(A), I(B))}{\varepsilon^{2} D^{2} / k^{2}} \\
= & \frac{\left(\begin{array}{c}
n-1 \\
k-1
\end{array}\right)^{2} o\left(1 / k^{2}\right)\left(D /\left(\begin{array}{c}
n-1 \\
k-1
\end{array}\right)\right)^{2}}{\varepsilon^{2} D^{2} / k^{2}}=o(1) .
\end{aligned}
$$

Предложение 9 доказано.

Из этого предложения следует тот факт, что для любого $\varepsilon>0$ при $n \rightarrow \infty$

$$
\mathbf{P}(\chi(\widetilde{G}) \leqslant(1+\varepsilon) D) \rightarrow 1 .
$$

Здесь использована оценка хроматического индекса (1).

Поскольку

$$
\chi(G(n, p)) \leqslant \chi\left(G^{\prime}\right)+\chi(\widetilde{G})
$$

отсюда получаем утверждение теоремы 3.

Автор благодарен своему научному руководителю А. А. Ирматову за ценные указания. 


\section{Список литературы}

1. Pippenger N., Spencer J., Asymptotic behavior of the chromatic index for hypergraphs. J. Comb. Theory (1989) 51, 24-42.

2. Будников Ю. А., Об асимптотическом поведении хроматического индекса случайных гиперграфов. Интеллектуальные системы (2007) 11, 343-360.

3. Ширяев А. Н., Вероятность. МЦНМО, Москва, 2004.

Статья поступила 12.02.2010. 